\title{
A Study on Appropriate Plant Diagram Synthesis for User-Suited HMI in Operating Control
}

\author{
Mieczyslaw Metzger and Grzegorz Polaków \\ Faculty of Automatic Control, Electronics and Computer Science \\ Silesian University of Technology, \\ Akademicka 16, 44-100 Gliwice, Poland \\ \{mieczyslaw.metzger,grzegorz.polakow\} @polsl.pl
}

\begin{abstract}
In this paper a study on appropriate plant diagram synthesis for user-suited HMI in operating control is presented. Discussion is based on the long-term personal experience and illustrated with excerpts of existing HMIs developed for research and industrial use. The HMI notion is defined for operating control and for operator training. The paper present three aspects of plant diagrams design. The first aspect deals with task-oriented usage of screen space for plant diagram and other GUI elements. Second aspect covers all methods of image creation for process diagrams, including photography, schematic diagrams, use of predefined normalised 3D graphical elements, and creative possibilities of 3D scene. The third aspect stresses capability of dynamic visualisation with the use of animated graphics.
\end{abstract}

Keywords: Usability of HMI for operating control, software engineering for user-suited HMI, plant diagram for GUI, visualisation, SCADA.

\section{Introduction}

Proper design of a plant diagram, according to the needs of a specific plant operator (or group of operators), is an important task, since the comfort of the plant operators' is the key issue influencing efficiency of their work, and implicitly accuracy of their decisions. Design of graphical user interfaces in, for example, computer entertainment industry, backed up by large finances due to potential profits, is nearly insignificant and hardly related to the importance of work and responsibilities of industrial process' operators.

Modern automation systems are equipped with operating panels implementing human-machine interface (HMI) for communication between a human operator and an industrial process being automated. HMI is the main part of the SCADA (Supervisory Control and Data Acquisition) application. The quality of the HMI determines a comfort of the human operator, and indirectly influences a productivity of one's work, therefore it should be designed in a user-suited way. A specific operator of a plant has his own partialities and habits, as humans, in general, have individual preferences for tools used in their everyday work and life. Therefore, a task of synthesising HMI suited to the specific human operator is worth considering. 
Publications and manuals for HMI synthesis deal mainly with the technical issues of GUI, like design of numerical control and indication fields, spatial layout of alert indicators, way of process trends presentation, etc. A very important part of the GUI, i.e. plant diagram, its design and synthesis, affecting human operator's perception and sense of aesthetics, is hard to normalise and should be suited to the specific user and tasks. In this paper a study on appropriate plant diagram synthesis for usersuited HMI in operating control is presented. Discussion is based on the long-term personal experience and illustrated with excerpts of existing HMIs developed for research and industrial use.

The paper is organised as follows. The next section presents much shortened overview of the related work in the bibliography. After that, the HMI notion is defined for the operating control and for the operator training. The following next three sections present three aspects of plant diagrams design. The first aspect deals with task-oriented usage of screen space for plant diagram and other GUI elements. Second aspect covers all methods of image creation for process diagrams, including photography, schematic diagrams, use of predefined normalised graphical elements, and creative possibilities of 3D graphics. Third aspect stresses capability of dynamic visualization with the use of animated graphics. Finally, a summary of major contributions is presented as concluding remarks.

\section{Related Work}

In this paper a discussion on synthesis of plant diagram for industrial HMI is presented. Hence, the shortened literature review here focuses on works dealing with cognitive and aesthetic aspects of industrial HMI. Recent developments in the area of usability in the human centred software engineering introduce some formal methods (see for example [1-5]), while fundamentals of human centred software engineering (HCSE) are well summarised in the books [6-7]. A special attention should be paid for the work of Seffah et.al. [5], in which 25 criterions for measuring usability are discussed. It should be also noticed, that there exist several attempts to standardisation, such as ISO/IS 9241, ISO/IS 13407, ISO/TR 18529, ISO 16071.

Different, slightly non-technical, approach for solving problems of the user interface design for effective human-computer interaction was presented in the books [89]. Ecological aspects of visual perception were discussed e.g. in [10], where it is stated that humans work more efficiently when interacting with three dimensional images as they are more analogous to the real world.

To mention particular ideas dedicated for synthesis of GUI being central part of HMI in industrial SCADA applications, earlier, more specialised publications must be referenced. It was 1986 when Norman in his note [11] introduced very important statement, that from the user's point of view it is the system's interface what is received as the actual system. A similar statement relating to simulators of industrial processes are found in [12].

In works [13-15] the investigation of colours and complexity of plant diagrams are presented. It is researched, how choice of colours and level of diagram's complexity influence the perception of the diagram. Wittenberg's work [15] additionally discusses three aspects of plant diagram design i.e. virtual process elements, task-oriented 
diagrams, and visualization of both goals and present condition. In our paper there are also three aspects of GUI synthesis discussed, from the other point of view.

\section{HMI for Operating Control and for Operator's Training}

After the revolution caused by common use of computers in nearly every sphere of everyday life, Human-Machine Interfaces (HMI) are used and required in many various applications, including very extensive and profitable area of computer entertainment. Before PCs spread and became popular, HMIs were used mainly in industrial systems requiring an operational control. Those systems (implemented as expensive specialised operating consoles) were designed by control instrumentation manufacturers and supplied by an investor as a part of whole industrial process. End-users had to accept the interfaces exactly as they were delivered.

Current wide availability of personal computers changed the situation. Operating consoles based on PC are cheap and readily obtainable. Diversity of the operating control software and the competition on the market makes it possible to easily and cheaply develop operating panels (nowadays called SCADA for Supervisory Control And Data Acquisition) according to the needs of a specific user. This paper focuses on proper choice and design of GUI being the main part of the HMI for the SCADA control operating system.

Fig. 1 symbolically presents the role of the GUI as perceived by the user of the system - in this case an operator of industrial process. As it was stated in the literature review, it is a GUI itself what is perceived by an operator as the system (see e.g. [11]). This statement is physically backed up by the fact, that in real plants, operating stations are located in the control room, which is spatially distant from the process. Until an emergency situation arises, there is no need for an operator to see and/or interact with the process, all the physical phenomena are represented for him with the GUI.

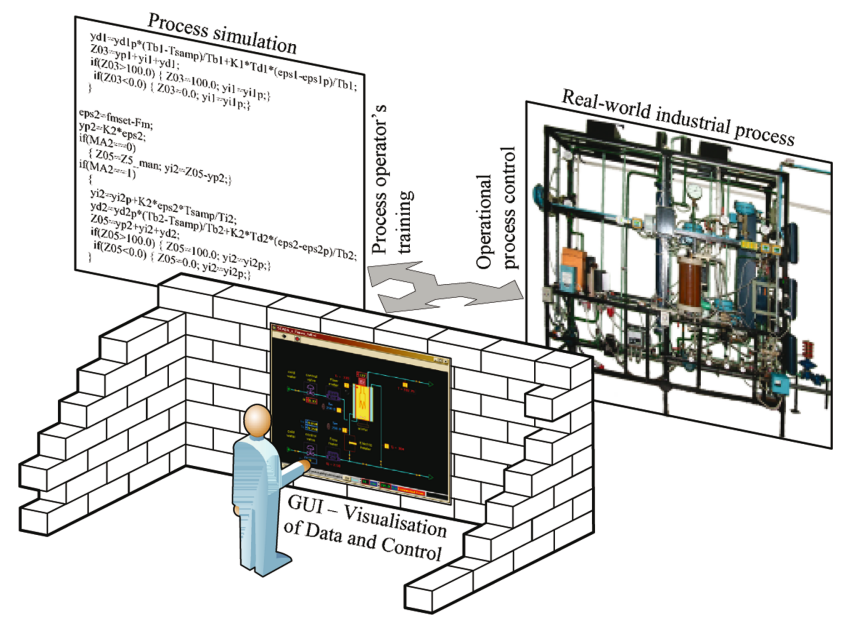

Fig. 1. General interpretation of GUI for process control 
A common availability of computers with a relatively high processing power enabled the possibility of creating real-time simulators of industrial processes, used to train potential system operators. As it was cited above, simulator (numerical software application) of an industrial process has to substitute the real process in such a way, that user believes that he interacts with the real process - see Fig. 1.

This implies the need for proper GUI, with which the user might interact exactly in the same way as in the case of real-world process. Hence, it should be noticed, that for proper training the same GUI should be designed both for the SCADA and for the simulator. In such case the operator is even unable to distinct if he controls the realworld process or the simulator at the time. Such possibilities are known and exploited in aerial and military applications for a long time, however it is the availability of computer hardware and software which enables those possibilities in civil domains. The discussion in this paper deals with such a GUI, which can be used both for operating control of a real process and for control of a real-time simulated process for operators training.

\section{Task-Oriented Usage of Screen Space for Plant Diagram}

Basing on twenty-year experience in the design of the HMI/GUI for industrial and research purposes, a following statement can be presented. In the majority of cases the usage of screen space depends on the task for which a particular GUI is designed.

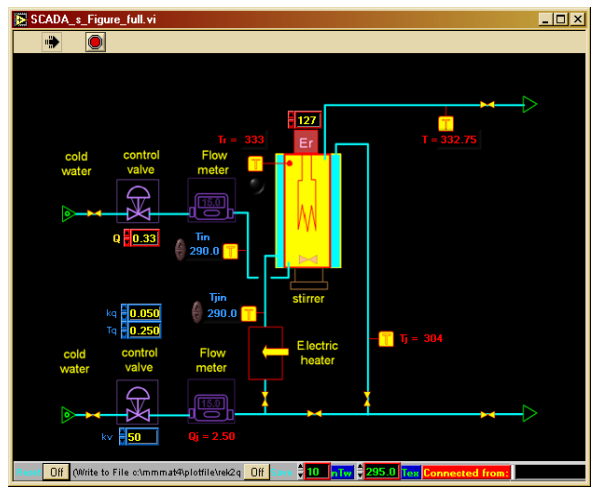

a)

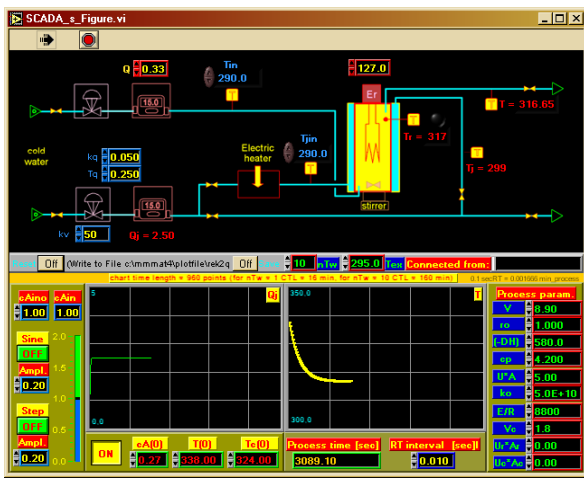

b)

Fig. 2. a) GUI for hybrid exothermic reactor during the normal production, b) GUI for hybrid exothermic reactor in the case of operating control for research experimentation

In Fig. 2a the GUI for operating control system of the industrial process during normal operation is presented. The discussion below is illustrated with excerpts of GUIs developed for our semi-industrial pilot plants that are presented in [16]. Specifically, in Fig. 2a the GUI for pilot hybrid exothermic reactor is shown. As in the typical industrial systems, the background of the diagram is black. During normal operation conditions, process diagram fills whole display space, because additional data (e.g. variable trends) are needed during exceptional conditions only, and typically the 
operator doesn't need them, as he is not conducting any researches. When these additional information are needed to be displayed, there can be temporarily placed in movable window in any preferable place of the diagram.

If process is being investigated, for example during start-up of the process, periodic checkout, and redesign of installation, there is a need to constantly watch and keep track of changes of variables' values and their trends. Because of this, an alternate GUI has to be designed for such conditions, in which the display space is divided into several parts in which there is a place for the plant diagram, charts of variables' trends, and increased number of controls supporting the investigations to be conducted. The diagram itself, in this case, requires scaling-down or trimming, so it fits to the limited display space. In Fig. $2 b$ the GUI is presented for the already introduced exothermic reactor, augmented with research capabilities.

\section{Plant Diagram Selection}

While there are both formal and informal attempts to standardisation of process diagrams, it seems that more user-centred approach could be possible, as colourpalette and aesthetics can modified on the fly for specific user needs. This could increase efficiency of process operator's work and improve accuracy of his decisions, according to the literature cited above.

Current state-of-art offers main four possibilities of process diagram creation technique (see Fig. 3):

a) Photograph of the real-world process;

b) Technical flowsheet;

c) 2D diagram composed of 3D and pseudo 3D elements;

d) Rendered full 3D scene.

For all the four stated techniques, research SCADA systems were developed (which are more complicated than typical exploitation SCADA). Basing on experiences gained during the synthesis of these applications and during their exploitation, following findings can be formulated.

The quickest and cheapest technique during creation and processing is photography. At the same time, its final effect is the least legible, because in the real installation there are many components, which are non-significant for the process operator. Controls and indicators are lost in the clutter, implying increased operator's reaction times. Nevertheless, photography, instead of simplification, shows the real looks of the process, which can be sometimes an advantage.

A technological chart based on a flow sheet uses the intentionally simplified diagram, displaying the most important process components only. Such design requires creativity and proper graphical tools. However, with no doubt, it is the most convenient and the most popular technique used in the practice. Software development environments for operating diagrams synthesis are usually supplied with a wide set of glyphs for the typical industrial instrumentation. 


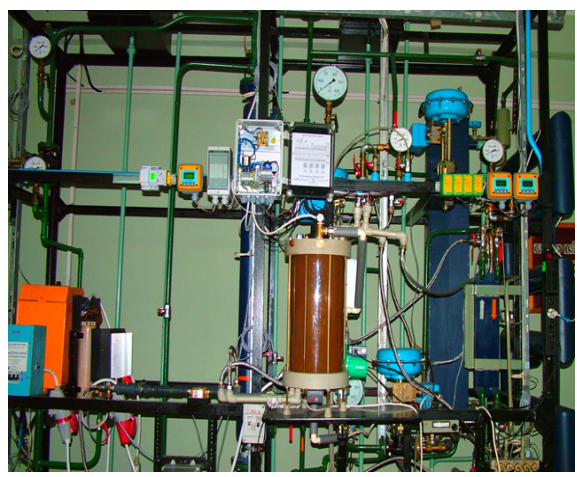

a)

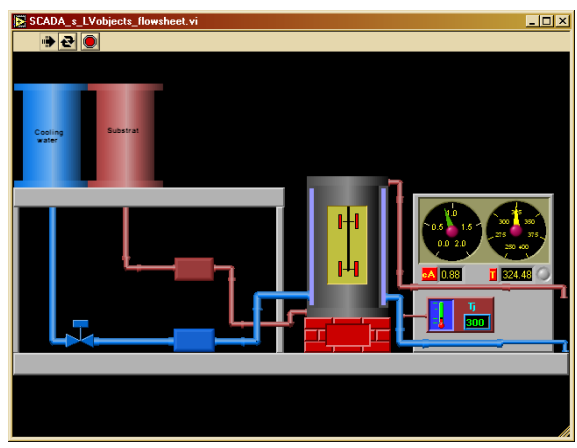

c)

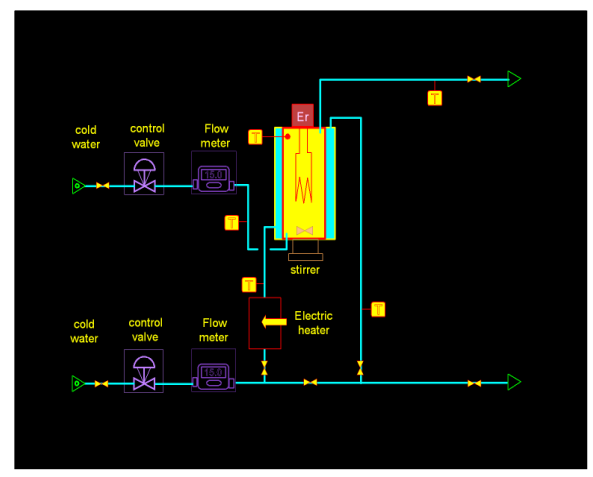

b)

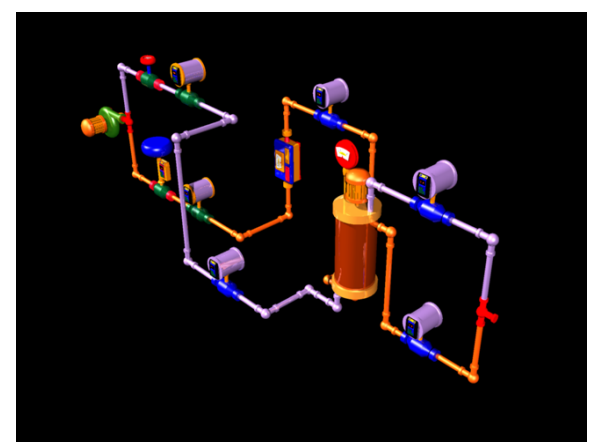

d)

Fig. 3. Most important possibilities of plant diagram creation: a) photograph, b) technical flowsheet, c) 2D diagram with 3D elements, d) rendered full 3D scene

The third technique requires a proper software environment capable of SCADA systems development, equipped with the $3 \mathrm{D}$ components, which could be placed in the diagram space. A representative example of such environment is the National Instruments LabVIEW [17], which was used to develop examples presented in this paper. Using the supplied libraries of components including numerical controls and indicators, an improved (when compared to the previous technique) diagram can be synthesised. Because the components are designed to be perceived as three dimensional by a human, the diagram is more readable and easier accepted by the operator. A significant shortcoming of this approach is the limited set of ready components supplied.

Three dimensional rendered scene is very attractive from visual point of view and contains (as two previous cases) only selected components of the system, which are important from operating control's point of view. However, this technique of diagram synthesis is the most difficult and complicated, as it requires expensive software tools, non typical for an industrial control. Time required for the development (modelling of the scene) is also significantly larger than in other techniques. It should be taken into 
account, that the instrumentation of the presented example is exceptionally simple when compared to industry-grade installation.

\section{Animation in Diagrams for Operating Control}

Animated graphics is still reluctantly accepted in the industrial applications, which is surprising as it can significantly increase capabilities of on screen visualisation.

In Fig. 4a the GUI for operating control of sequencing reactor in wastewater treatment process is presented. The key tasks of control in this process is turning the stirring and aeration on and off adequately. Because of this, proper diagram elements are animated when corresponding action is in progress. Such animated elements are not provided as standard components and they have to be developed specifically for the task, however gained effect and productivity increase are worth the effort.

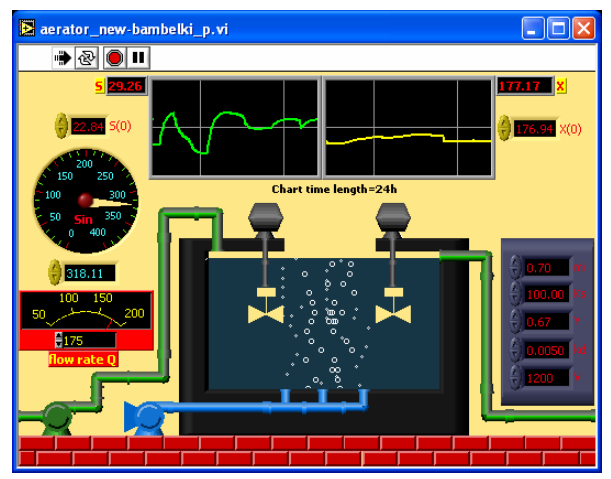

a)

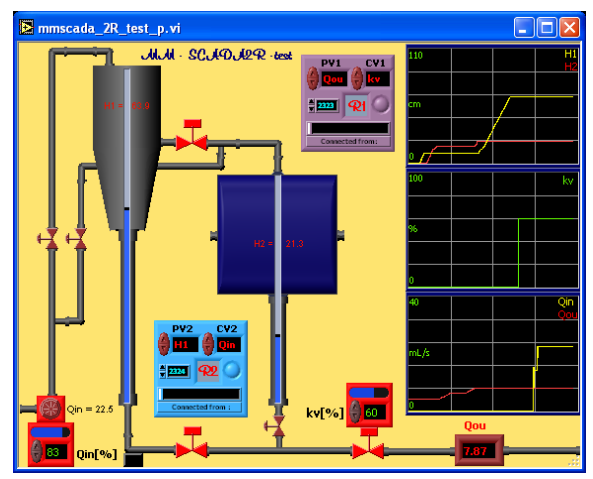

b)

Fig. 4. a) GUI for sequenced biotechnological process with animated elements (switching-on and switching-off paddle mixers and floating bubbles of air), b) GUI for hydraulic process with changeable architecture (only pipes which are open at the moment are displayed in the diagram)

Some industrial processes are designed to have changeable structure of components connection, for example by cutting a flow of a liquid in a pipe. To enhance the visualisation, this modified structure may by presented in the GUI. For example, pipes closed with valves may disappear from the diagram completely. Fig. 4b presents such a GUI. For illustrative purposes, all the pipes are shown, but during the runtime only the current configuration of the piping is displayed.

\section{Concluding Remarks}

In this paper a study on appropriate plant diagram synthesis for user-suited HMI in operating control is presented. Discussion is based on the long-term personal experience and illustrated with excerpts of existing HMIs developed for research and industrial use. 
After defining the HMI notion for the operating control and for the operator training, the paper describes three areas of the plant diagrams design process. The proper usage of a screen space for the plant diagram and other HMI elements is described. Then, four methods of image creation for process diagrams are depicted, i.e. photography, schematic 2D diagrams, pseudo-3D diagrams composed of predefined graphical elements, and ray-traced full 3D scene. At last, dynamic visualisation capabilities of the animated graphics are outlined.

The approach proposed here is not opposed to the one based on the formal methods, but it rather complements that methods with the point of view of industrial HMIs developers. The practice shows that users often prefer specific solutions, and their preferences are not based on any objective measurable principles, but are extremely subjective. Enabling the user to choose the variant of the graphical layout of the interface on his own is therefore highly desirable. The presented survey of methods can be used as a start point for the process of user-suited HMI design.

Acknowledgements. This work was supported by the Polish Ministry of Scientific Research and Education.

\section{References}

1. Vicente, K.J., Rasmussen, J.: Ecological Interface Design: Theoretical foundations. IEEE Transactions on Systems, Man and Cybernetics 22, 589-606 (1992)

2. Paterno, F.: Formal reasoning about dialogue properties with automatic support. Interacting with computers 9, 173-196 (1997)

3. Jamieson, G.A., Vicente, K.J.: Ecological interface design for petrochemical applications: supporting operator adaptation, continuous learning, and distributed, collaborative work. Computers and Chemical Engineering 25, 1055-1074 (2001)

4. Seffah, A., Forbrig, P., Javahery, H.: Multi-devices "Multiple" user interfaces: development models and research opportunities. The Journal of Systems and Software 73, 287 300 (2004)

5. Seffah, A., Donyaee, M., Kline, R.B., Padda, H.K.: Usability measurement and metrics: A consolidated model. Software Qual. J. 14, 159-178 (2006)

6. Burns, C.M., Hajdukiewicz, J.R.: Ecological Interface Design. CRC Press, Boca Raton (2004)

7. Seffah, A., Gulliksen, J., Desmarais, M.C.: Human-Centered Software Engineering - Integrating Usability in the Software Development Lifecycle. Springer, Heidelberg (2005)

8. Rasmussen, J.: Information processing and human-machine interaction: An approach to cognitive engineering. North-Holland, Amsterdam (1986)

9. Shneiderman, B.: Designing the user interface - strategies for effective Human-Computer Interaction. Addison-Wesley, Reading (1998)

10. Gibson, J.J.: The ecological approach to visual perception. Houghton Miffin (1979)

11. Norman, D.A.: Cognitive Engineering. In: User Centered System Design: New Perspectives On Human-Computer Interaction, p. 61. Erlbaum, Hillsdale (1986)

12. Metzger, M.: A new concept of industrial process simulation - cybernetic approach using distributed access control schemes. SAMS 15, 185-202 (1994)

13. Inverso, D., Sokoll, R.: Optimum Human-Interface. Control Engineering, 93-98 (September 1997) 
14. Johnson, D.: Conveing understandable process information to an operator requires more than dazzling HMI graphics in real time. Control Engineering, 80-88 (September 1997)

15. Wittenberg, C.: A pictorial human-computer interface concept for supervisory control. Control Engineering Practice 7, 865-878 (2004)

16. Metzger, M., Polaków, G.: Holonic Multiagent-Based System for Distributed Control of Semi-industrial Pilot Plants. In: Mařík, V., Vyatkin, V., Colombo, A.W. (eds.) HoloMAS 2007. LNCS (LNAI), vol. 4659, pp. 338-347. Springer, Heidelberg (2007)

17. Official National Instruments LabVIEW website, http://www.ni.com/labview/ 